\title{
NUMERICAL MODELLING OF CAVITATION: VALIDATION AND PARAMETRIC STUDIES
}

\author{
X. Margot *, S. Hoyas, A. Gil and S. Patouna \\ CMT - Motores Térmicos, Universidad Politécnica de Valencia, Camino de Vera S/N \\ 46022 Valencia, Spain \\ *E-Mail: xmargot@mot.upv.es (Corresponding Author)
}

\begin{abstract}
The objective of the present work is to investigate numerically the 3D flow within diesel injectorlike geometries using a cavitation model implemented in a commercial CFD code. A comprehensive study of various numerical parameters is performed which can subsequently be used to simulate cavitation under realistic diesel engine conditions. Numerical predictions were performed on a throttle channel at different operating conditions, with and without cavitation, and compared to available experimental measurements. Overall, it was found that the cavitation model was able to predict the onset of cavitation. Satisfactory agreement was found in both the injection rate and the occurrence of choked flow conditions when compared with experiments.
\end{abstract}

Keywords: cavitation, injection nozzle, modelling, choked flow, two-phase flow

\section{INTRODUCTION}

The understanding of the internal flow dynamics phenomena occurring in an injector is important due to their strong association with the atomisation process and consequently with the combustion process (Balasubramanyam et al., 2010). However, observing the flow in diesel injectors is very difficult as they are characterised by high pressure injections through very small nozzles with diameters of around 100 microns or less. In addition, a decrease in pressure below a critical level leads to cavitation (Berwerk, 1959; Nurick, 1976; Arai et al., 1985), which adds another level of complexity to the problem (Soteriou et al., 1995).

In most cases cavitation is undesirable since it causes performance loss, material damage, vibrations and noise (Knapp et al.,1970; Brennen, 1995). On the contrary, in engine fuel injection systems, and particularly in diesel injectors, it is expected that nozzle cavitation enhances jet turbulence, which in turn promotes fuel atomization (Roth et al., 2002; He and Ruiz, 1995). It is therefore of great importance to study the cavitation phenomena inside the nozzle, to understand cavitation issues and to find out how it is correlated to the flow characteristics in the combustion chamber.

For the accurate prediction of spray atomization, identification of the parameters describing the fuel jet leaving the nozzle is essential. One of the first comprehensive experimental studies on cavitation structures in diesel injector-like geometries was performed by Winklhofer et al. (2001). Other experiments have been performed in scaled-up nozzles to observe the nozzle internal flow (Hiroyasu et al., 1991; Chaves et al., 1995; Soteriou et al., 1995). However, it was concluded that cavitation does not scale up, and therefore actual-size experiments are needed to depict the cavitating flow behaviour (Arcoumanis et al., 2000). Due to the difficulty of directly measuring the characteristics of the two-phase flow in very small orifices such as found in automotive engine applications, the use of numerical simulations (Bierbrauer and Zhu, 2008; Christafakis and Tsangaris, 2008) is very useful in order to understand the flow features inside and at the exit of the injector nozzle.

The objective of the present study is to investigate numerically the effects of various parameters on the flow characteristics inside an injector-like nozzle, as well as at the exit, to be able subsequently to apply the results of this study to analyse the flow in real diesel injectors operating conditions (above $100 \mathrm{Mpa}$ ). For this, a cavitation model based on bubble growth theory (Rayleigh, 1917) was used, as implemented in a commercial code (STAR-CD, 2008). Extensive validation was performed using available two-phase flow experimental data (Winklhofer et al., 2001), obtained with a transparent throttle channel flow. The paper starts (section 2) with a mathematical description of the models implemented in the simulations. In section 3 , the predicted results are compared with the measurements of the vapour field distribution, pressure field and velocity

Received: 17 Nov. 2010; Revised: 12 Jul. 2011; Accepted: 1 Aug. 2011 
profiles. The paper ends (section 4) with a summary of the most important conclusions.

\section{NUMERICAL MODEL}

\subsection{Computation domain and grid independence results}

For the validation and parametric studies performed within this work, the injector-like throttle channel geometry $\mathrm{J}$ of Winklhofer et al. (2001) was considered. Since this geometry is symmetric (Fig. 1), only half of the geometry was calculated by imposing symmetry boundary conditions along the axis boundary. Constant pressure boundary conditions were set at the inlet and outlet and the no slip boundary condition was used at the wall. Although the size of the channel is substantially larger than current diesel injector orifices, this geometry has been used as a reference in the literature (Shi and Arafin 2010; Som et al., 2010) and can therefore be used to validate the model. The calculations were performed taking into account the roundness of the nozzle entrance $(\mathrm{r}=20 \mu \mathrm{m})$ and the threedimensional nature of the flow. The cross-section of the nozzle is square (width, Y-axis $=299 \mu \mathrm{m}$ and thickness, $Z$-axis $=300 \mu \mathrm{m})$ and the length is $1000 \mu \mathrm{m}$ (X-axis), as shown in Fig. 1.

The results of CFD calculations are known to be affected by the resolution of the computational mesh, especially in the regions of high gradients. In order to verify the grid independence of the solution, different adaptive refinements were performed. To start with, the calculations were realised with the mesh resolution shown in Fig. 2 (121820 cells) with average cell size of $1-3 \mu \mathrm{m}$. Fig. 3 shows the refined zones, which resulted from the refinement process based on pressure gradients and velocity magnitude gradients, respectively, yielding a total cell number of 207094. It is seen that with the pressure-gradients method, the mesh was refined at the inlet corner, while with the velocity magnitude gradients it was refined in the upper part of the nozzle. However in terms of injection rate the differences between the meshes are not significant, maximum $3 \%$ (see Table 1). Nevertheless, the mesh adopted for the calculations was the mesh with a local refinement at the nozzle entry to capture the large pressure and velocity gradients in this region related with the onset of cavitation.

\subsection{Cavitation modelling approach}

In the current study, a commercial code (STARCD 2008) is used for the flow and cavitation

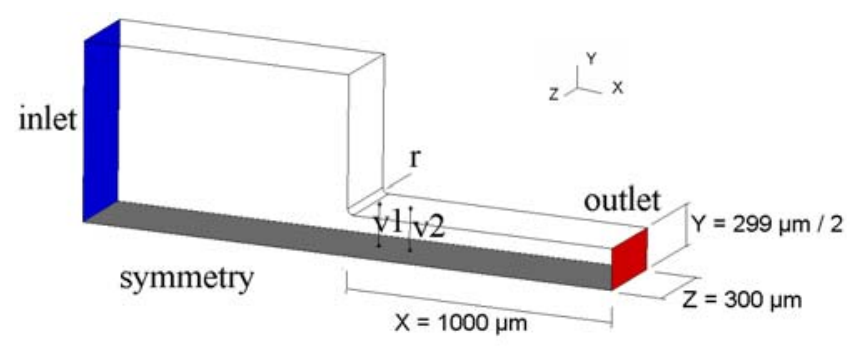

Fig. 1 Geometry of computation domain and boundary conditions (v1: $53 \mu \mathrm{m}$ inside the throttle hole, v2: $170 \mu \mathrm{m}$ inside the throttle hole).

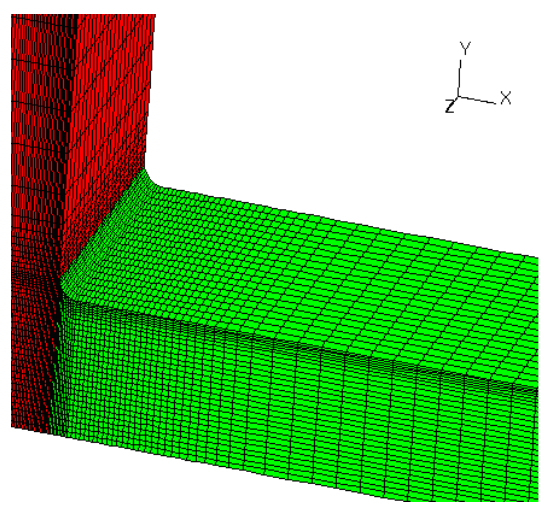

Fig. 2 Visualization of mesh distribution.

(a)

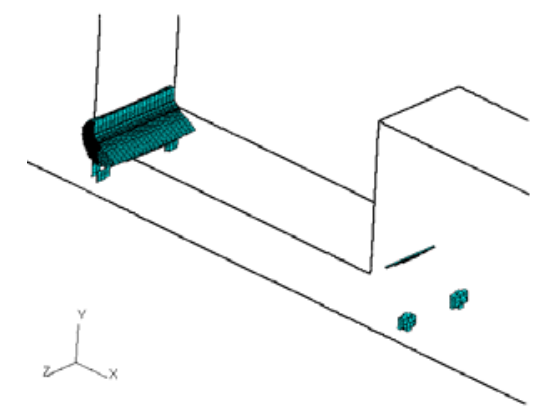

(b)

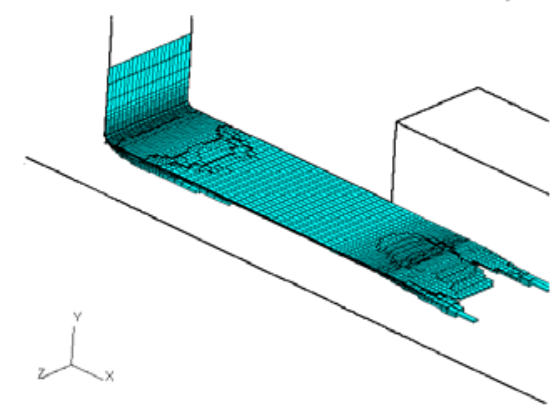

Fig. 3 Refined locations by (a) pressureand (b) velocity magnitude.

Table 1 Injection rate results ( $\mathrm{g} / \mathrm{s})$ obtained by different mesh resolutions.

\begin{tabular}{|l|c|c|c|}
\hline & $\begin{array}{l}\text { Coarse } \\
\text { mesh }\end{array}$ & $\begin{array}{l}\text { Refined by } \\
\text { pressure }\end{array}$ & $\begin{array}{l}\text { Refined by } \\
\text { velocity }\end{array}$ \\
\hline $\begin{array}{l}\text { Injection rate } \\
(\mathrm{g} / \mathrm{s})\end{array}$ & 8.26 & 8.14 & 8.04 \\
\hline
\end{tabular}


prediction. The basic assumptions of the cavitation model implemented in the code are the following:

- There are cavitation seed bubbles present in the liquid, homogeneously distributed and of equal size; the initial seed radius is one of the model parameters and needs to be specified.

- The number of seed bubbles per unit volume of liquid is constant and is also a model parameter to be specified.

- All bubbles present in a control volume at any time are spherical and of the same size; however the bubble radius changes with time, as expressed by equation 3 below.

- Both liquid and vapour densities are constant.

- The density ratio of vapour to liquid is small, $<<1$.

- There is no slip between bubbles and liquid.

The liquid and the vapour phase are treated as a mixture with a transport equation for the volume fraction of vapour phase, which is computed using the following equation:

$\frac{\partial a_{v}}{\partial t}+\nabla\left(a_{v} u\right)=S a_{v}$

where subscript $v$ denotes the vapour phase, $u$ is the flow velocity and $S a_{v}$ is the cavitation induced mass source/sink term of volume fraction $a_{v}$, which is calculated by a model based on bubble growth theory (Rayleigh, 1917).

$S a_{v}=\frac{4 \pi R^{2} n_{o}}{1+\left(4 \pi R^{3} / 3\right) n_{o}} \frac{d R}{d t}$

The bubble radius $R$ changes according to the local pressure $p$ around the bubble, as bubbles move through the solution domain $\left(n_{o}\right.$ is prescribed number of spherical bubbles of radius $\mathrm{R}$ within a unit volume of liquid). The rate of change of a bubble radius along its path (the bubble growth velocity) is estimated using the following equation, which is a simplification of the more general Rayleigh-Plesset equation.

$\frac{d R}{d t}=\operatorname{sign}\left(p_{v}-p\right) \sqrt{\frac{2\left|p_{v}-p\right|}{3 \rho_{1}}}$

where $p_{v}$ is the saturation pressure $\left(p_{v}=3000 \mathrm{~Pa}\right.$ in this study) and $\rho_{l}$ is the liquid density. The critical pressure in this study is associated with the saturation pressure in liquid to explain the onset of cavitation. Recent work of cavitation takes into account the effect of the turbulent shear stress on the critical pressure (Martynov, 2005) based on the concept of stress-induced cavitation in flows of high-viscosity fluids (Joseph, 1998). In his theoretical analysis, Joseph argued that the liquid may rupture when the maximum of the principal component of the stress tensor overcomes the vapour pressure in liquid. This criterion determines the onset of cavitation in the flowing liquid. Although the approach is very novel, it seems rather impractical since it implies a stress analysis in the whole flow field in order to apply the proposed criterion. The model approximates the rates of evaporation and condensation as linear functions of pressure and neglects non-condensable gases. The effect of liquid surface tension is not taken into account. The seed radius was set to $1 \mathrm{E}-06 \mathrm{~m}$ and the nuclear number density to $1 \mathrm{E}+14 \mathrm{~m}^{-3}$, in agreement with values proposed in the literature for real-size nozzle simulations (Yuan et al., 2000; Yuan and Schnerr, 2001). Further details concerning the basic differential conservation equations and the turbulence model can be found in (Margot et al., 2010; Payri et al., 2009).

\subsection{Turbulence modelling approach}

The flow inside the injector-like nozzle considered in this work is clearly turbulent since its Reynolds number is about 12000 . Hence, a turbulence model is required for the flow simulation. In this study, a reference flow configuration with the $\mathrm{k}-\varepsilon / \mathrm{low}$ Reynolds/hybrid turbulence model was first defined and calculated (see Table 2). A turbulence model study was then performed, mainly with variants of the k- $\varepsilon$ and the $\mathrm{k}-\omega$ turbulence models to check their influence on the predicted flow field from an engineering point of view (see Table 2). For this study, all other parameters were kept as in the reference case. The results obtained with the different turbulence approaches tested are illustrated in Fig. 4, in terms of velocity profiles at cavitating and non cavitating conditions. These are extracted at positions where the experimental data from Winklhofer et al (2001) are available. It is seen that by using the different models, for a certain mesh resolution, the velocity profiles show slight differences, mainly about $2 \%$. It was also found that the maximum difference with the various turbulence models is about $2 \%$ in terms of injection rate, which is the key parameter for the validation. In view of these results, it was finally decided to use the $k-\varepsilon /$ low Reynolds number 
Table 2 Reference configuration (highlighted) used as basis for parameters variation study and definition of parameters varied: turbulence model, time step, solution algorithm, seed radius and nucleus number.

\begin{tabular}{|c|c|c|c|c|}
\hline \multicolumn{5}{|c|}{ Reference configuration used for calculations } \\
\hline k- $\varepsilon /$ low Reynolds/hybrid & $\begin{array}{l}\text { Medium time } \\
\text { step }(1.0 \mathrm{E}-06)\end{array}$ & $\begin{array}{l}\text { Conjugate } \\
\text { Gradient }\end{array}$ & $1.0 \mathrm{E}-06$ & $1.0 \mathrm{E}+14$ \\
\hline \multicolumn{5}{|c|}{ Parameters varied (one at a time based on reference configuration) } \\
\hline $\begin{array}{l}\text { Turbulence modelling approach/ wall } \\
\text { treatment }\end{array}$ & Time step & $\begin{array}{c}\text { Solution } \\
\text { algorithm }\end{array}$ & $\begin{array}{c}\text { Seed } \\
\text { radius }\end{array}$ & $\begin{array}{l}\text { Nucleus } \\
\text { number }\end{array}$ \\
\hline k- $\varepsilon /$ high Reynolds/standard & \multirow{6}{*}{$\begin{array}{l}\text { Small time step } \\
(5.0 \mathrm{E}-07) \\
\text { Large time step } \\
(2.0 \mathrm{E}-06)\end{array}$} & \multirow{6}{*}{$\begin{array}{l}\text { Algebraic } \\
\text { Multigrid }\end{array}$} & \multirow{6}{*}{$\begin{array}{l}1.0 \mathrm{E}-05 \\
5.0 \mathrm{E}-05\end{array}$} & \multirow{6}{*}{$\begin{array}{l}1.0 \mathrm{E}+12 \\
1.0 \mathrm{E}+16\end{array}$} \\
\hline k- $\omega$ SST/low Reynolds/hybrid & & & & \\
\hline k- $\omega$ SST/high Reynolds/standard & & & & \\
\hline k- $\omega$ standard/low Reynolds/hybrid & & & & \\
\hline k- $\omega$ standard/high Reynolds/standard & & & & \\
\hline $\mathrm{k}-\varepsilon \mathrm{RNG} / \mathrm{standard}$ & & & & \\
\hline
\end{tabular}

(a)
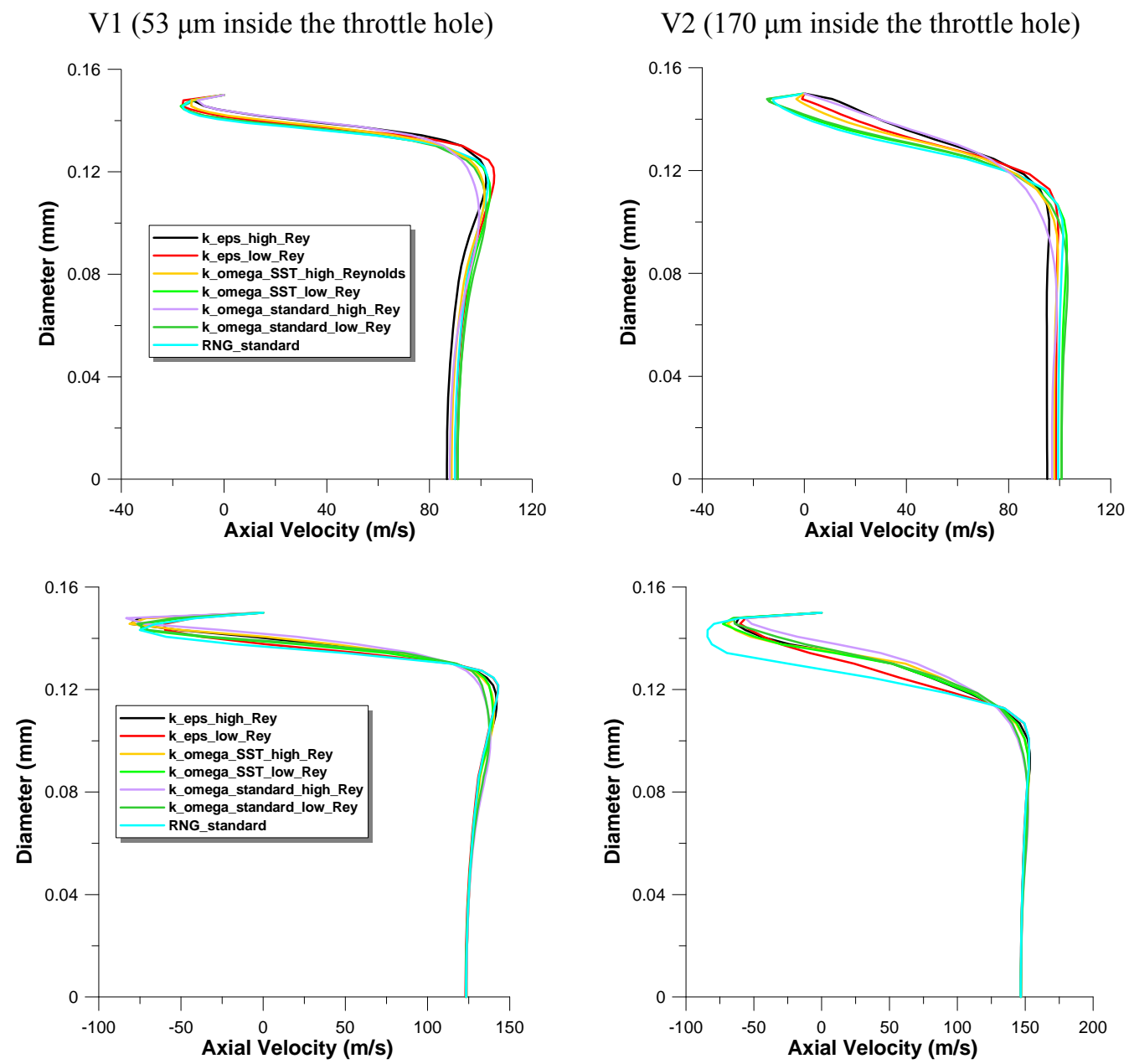

Fig. 4 Predicted velocity profiles obtained with different turbulence model approaches in position v1 and v2 (a) at condition without cavitation ( $\Delta \mathrm{p}=41$ bar) and (b) at choked flow conditions ( $\Delta \mathrm{p}=85$ bar).

turbulence model with hybrid near wall treatment for closure of the equations. This model seemed to be the most appropriate for the range of Reynolds numbers considered, indicative of transitional rather than fully turbulent flow, as well as a good compromise between approximation to experimental results in terms of injection rate and solution cost. In addition, the hybrid near wall treatment provides valid boundary conditions for a wide range of near-wall mesh densities, which ensures independence of the $\mathrm{y}^{+}$value, and it is better adapted to this type of flow than the standard wall function approximations, generally used for high Reynolds number models. 


\subsection{Computational method}

The solver is based on the finite volume approach and the iterative process has been performed with the SIMPLE algorithm. Although inlet and outlet pressures are constant, the modelling of the cavitation phenomenon itself is transient. The cavitating flow has to be computed in a timemarching manner, even if the final solution is steady-state. In the rest of the text, this approach will be termed "quasi-steady state" calculations. In order to accelerate convergence and ensure stability of the calculations, the solution was converged to steady state before connecting the cavitation model and performing the corresponding time-dependent calculations. To analyse the influence of the time-step on the solution, several calculations were performed, with different values of the time-step (5.0E-07; $1.0 \mathrm{E}-06 ; 2.0 \mathrm{E}-06)$ and all other parameters as in the reference configuration (see Table 2). For the convective flux approximation, a second order scheme (MARS) for the momentum equations and a first order scheme (UD) for the $k-\varepsilon$ equations was used. The working fluid was diesel fuel with properties as given in Table 3a. This reference configuration (see highlighted lines of

Table 3 Fluid properties used: (a) present calculations and (b) Peng Kärrholm et al., (2007).

\begin{tabular}{lll|ll}
\hline \multicolumn{3}{c|}{ a } & \multicolumn{2}{c}{ b } \\
\hline & Liquid & Vapor & Liquid & Vapor \\
\hline $\begin{array}{l}\text { Density } \\
\left(\mathrm{Kg} / \mathrm{m}^{3}\right)\end{array}$ & 828 & 0.025 & 832 & 0.1361 \\
$\begin{array}{l}\text { Viscosity } \\
(\mathrm{kg} / \mathrm{ms})\end{array}$ & $2.14 \times 10^{-3}$ & $1 \times 10^{-5}$ & $6.5 \times 10^{-3}$ & $5.953 \times 10^{-6}$ \\
\hline
\end{tabular}

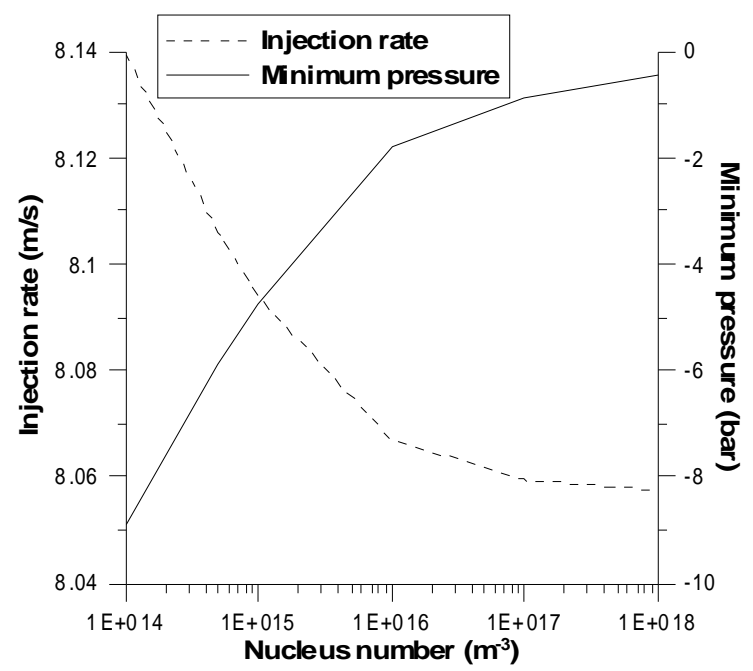

Fig. 5 Injection rate and minimum pressure as a function of nucleus number density $(\Delta \mathrm{p}=85$ bar).
Table 2 and Table 4) was implemented and used to obtain the results presented in this paper. Some additional parameters that may influence the solution, referring to both the solver and the cavitation model itself, were each varied in turn, maintaining the others of the reference configuration constant, as shown in Table 2. From these parametric studies, it may be concluded on the one hand that the effect on the injection rate of varying only the time-step, or the seed radius, or changing the solution algorithm is negligible. On the other hand, a change of the discretization scheme (see Table 4) or a variation of the nucleus number density (see Fig. 5) has slightly more influence, resulting in a variation of the injection rate of up to $1.5 \%(\Delta \mathrm{p}=85$ bar $)$.

The computational (CPU) time of the singlephase runs to reach the steady-state calculation was of the order of $1 \mathrm{~h}$ per calculation on a core of an Intel QuadCore Xeon CPU @ 2.00 GHZ, while the CPU cost of the quasi-steady state cavitation runs was of the order $1 \mathrm{~min}$ per time step.

Table 4 Combinations of considered discretization schemes and injection rates (UD-upwind differencing- $1^{\text {st }}$ order, CD-Central Differencing- $2^{\text {nd }}$ order, MARS-Monotone Advection and Reconstruction Scheme- $2^{\text {nd }}$ order), where highlighted schemes are the reference configuration.

\begin{tabular}{cccc}
\hline Momentum & Turbulence & Cavitation & $\begin{array}{c}\text { Injection } \\
\text { rate (g/s) }\end{array}$ \\
\hline MARS & UD & UD & 8.14 \\
MARS & CD & UD & 8.20 \\
MARS & UD & CD & 8.14 \\
UD & UD & UD & 8.24 \\
CD & UD & UD & 8.16 \\
\hline
\end{tabular}

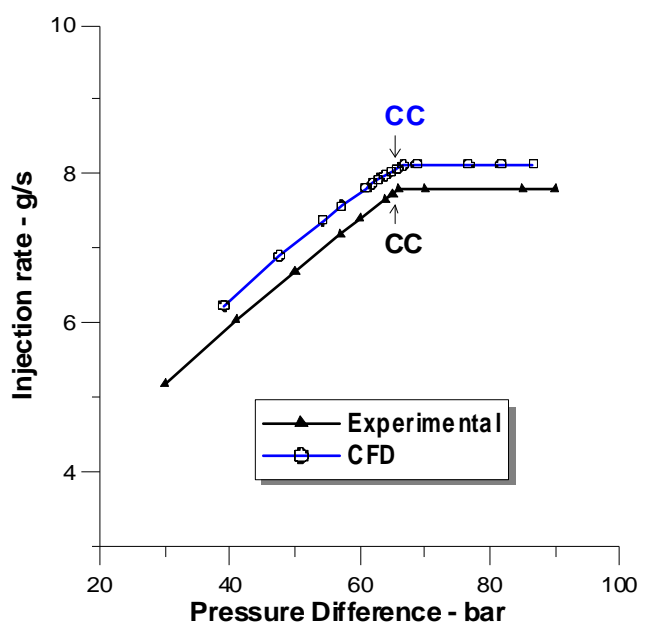

Fig. 6 Experimental data from Winklhofer et al. (2001) and predicted injection rate plotted versus the pressure difference, where $\mathrm{CC}$ are critical conditions. 


\section{CALCULATION RESULTS AND DISCUSSION}

\subsection{Validation cases}

As mentioned above, the optical and hydraulic characterization of a transparent throttle channel flow (geometry J) carried out by Winklhofer et al. (2001) has been used in this work as a reference to validate the CFD model. As in the experiments, the injection pressure has been kept constant (100 bar), while backpressure has been varied to get the corresponding pressure drop, and thus study the flow in non cavitating and cavitating conditions.

\subsubsection{Injection rate results and cavitation field}

In Fig. 6 the results of the injection rate of the test case at different pressure drops is compared with the measurements. The study is aimed at showing the ability of the model to describe variations in the length of the vapour region with the cavitation number, rather than at providing exact validation of the model, since this would require more specific experimental information about the cavitating flow. As seen in Fig. 6, the main deviation with respect to the experimental data is on the critical conditions, above which the mass flow becomes choked. The preliminary calculated results show that the mass flow collapses when the outlet pressure is 30 bar (i.e. $\Delta p=70$ bar) instead of 35 bar (i.e. $\Delta \mathrm{p}=65$ bar) as observed experimentally. The total pressure in the simulation cases is reduced by the dynamic

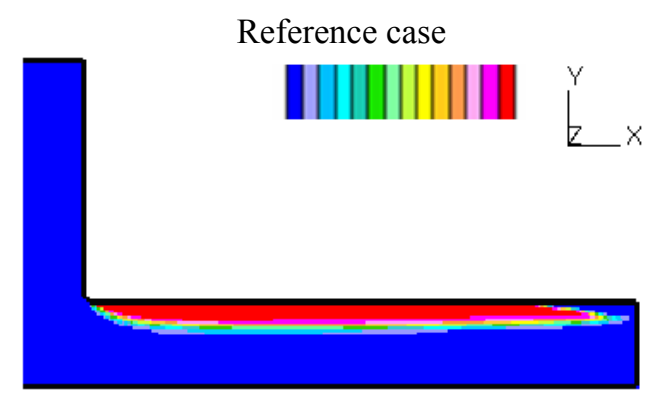

pressure at the inlet, which represents a pressure loss at the entrance. The pressure difference in the predicted cases is estimated by taking into account this inlet loss.

The injection rate predicted results are overestimated by about $4 \%$ at increasing cavitating conditions. However, this deviation is not critical for the further analysis of this case study. In fact, the slight over-prediction may be linked to the uncertainties in the values of liquid viscosity. Indeed, it was found that the effect of liquid viscosity can have a significant influence on the amount of cavitation. Considering the lack of experimental values, additional calculations were made with fuel properties found in the literature (Peng Kärrholm et al., 2007) in order to check the effect of viscosity (see Table 3). It was observed that there was less generation of volume fraction of vapor with higher viscosity (see Fig. 7). Higher viscosity leads to lower dynamic pressure and thus yields higher absolute pressure values and less cavitation in accordance with Shi and Arafin (2010). However, as a general trend, the cavitation model predicts a reduction of the nozzle mass flow rate with increasing cavitation intensity, which is in agreement with the measurements.

Cavitation appears in the nozzle hole entrance due to the abrupt change of the flow direction associated with large pressure drop and very high fuel velocities. With decreasing back pressure, it expands up to the exit with major volume fraction of vapour at the upper and lower part of the nozzle (Fig. 8).

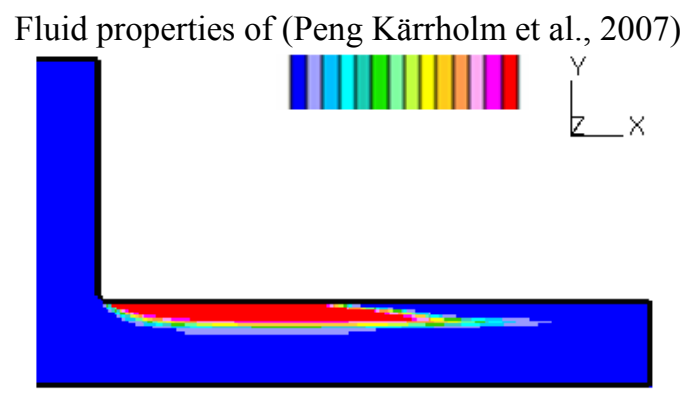

Fig. 7 Volume fraction of vapour distribution with different fluid properties at longitudinal middle plane, colour scale: $0-1$.
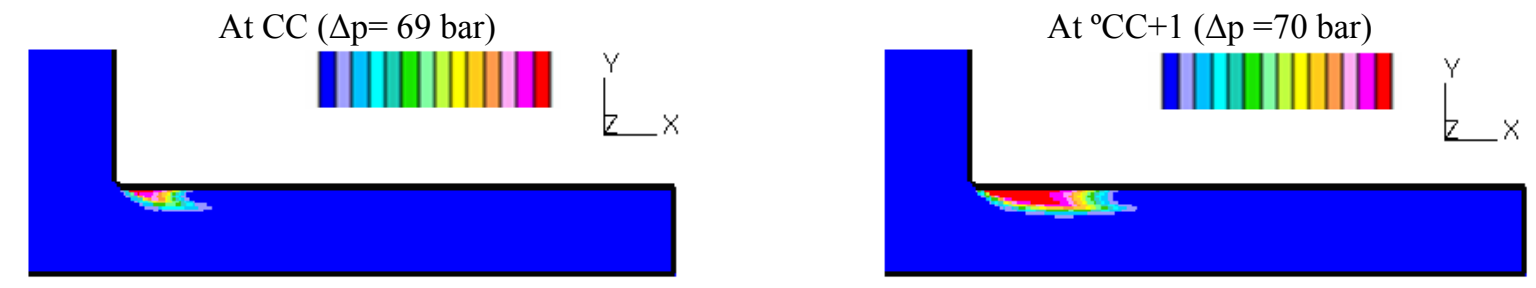

Fig. 8 Predicted volume fraction of vapour fields around critical cavitation at longitudinal middle plane, colour scale: 0-1. 
Preliminary calculations using different density numbers of cavitation bubbles have shown that even using the highest value of those recommended (in the range of $1 \mathrm{E}+11 \mathrm{~m}^{-3}-1 \mathrm{E}+14$ $\mathrm{m}^{-3}$ ) for high-pressure, high-velocity systems, the amount of vapour observed in experiments could not be predicted with accuracy, as only a few cells near the nozzle inlet corner were filled with vapour (Fig. 8). This discrepancy can be explained by the influence of the turbulence model and the fluid viscosity, as mentioned above. However, the cavitation field in the predicted cases grows significantly just by increasing the pressure drop from critical cavitation (CC) to $\mathrm{CC}$ +1 bar (see Fig. 8), as also observed in the experiment.

\subsubsection{Velocity profiles}

Velocity profiles are shown in Fig. 9 for operating conditions without cavitation $(\Delta \mathrm{p}=41$ bar), with moderate cavitation $(\Delta \mathrm{p}=70$ bar $)$ and at choked flow conditions $(\Delta \mathrm{p}=85$ bar). The velocity profiles are extracted downstream of the throttle entrance at the position $\mathrm{v} 1(53 \mu \mathrm{m}$ inside the throttle hole) and v2 (170 $\mu \mathrm{m}$ inside the throttle hole). These are essentially located in the main part of the flow contraction zone (Fig. 1). The measured velocity profiles described in Winklhofer et al. (2001) refer to geometry U (301 $\mu \mathrm{m}$ inlet and $284 \mu \mathrm{m}$ outlet width). Hence, only a qualitative comparison can be made with respect to geometry $\mathrm{J}$ studied here. In agreement with Winklhofer et al. (2001), at position v1 the velocity peaks near the shear layer and shows a minimum in the channel center. This profile is also present at higher pressure drops where the velocity peak near the cavitation region significantly increases. According to Winklhofer et al. (2001), at position v2 the velocity profile changes with respect to that of position $\mathrm{v} 1$, with the maximum velocities observed near the boundary of the cavitation region and also in the channel center. The latter, however, is not observed in the calculation: the velocity profiles are flat at the center, until affected by the cavitation zone.

The velocity profiles also show reverse flow near the wall in the hole entrance, thus indicating that the flow separates in this region. According to the observations by Winklhofer et al. (2001), at a higher pressure drop the cavitation seems to initiate in the separated shear layer. With increasing formation of cavitation, the low pressure recirculation area fills with vapour. The same is observed in the numerical solution of the
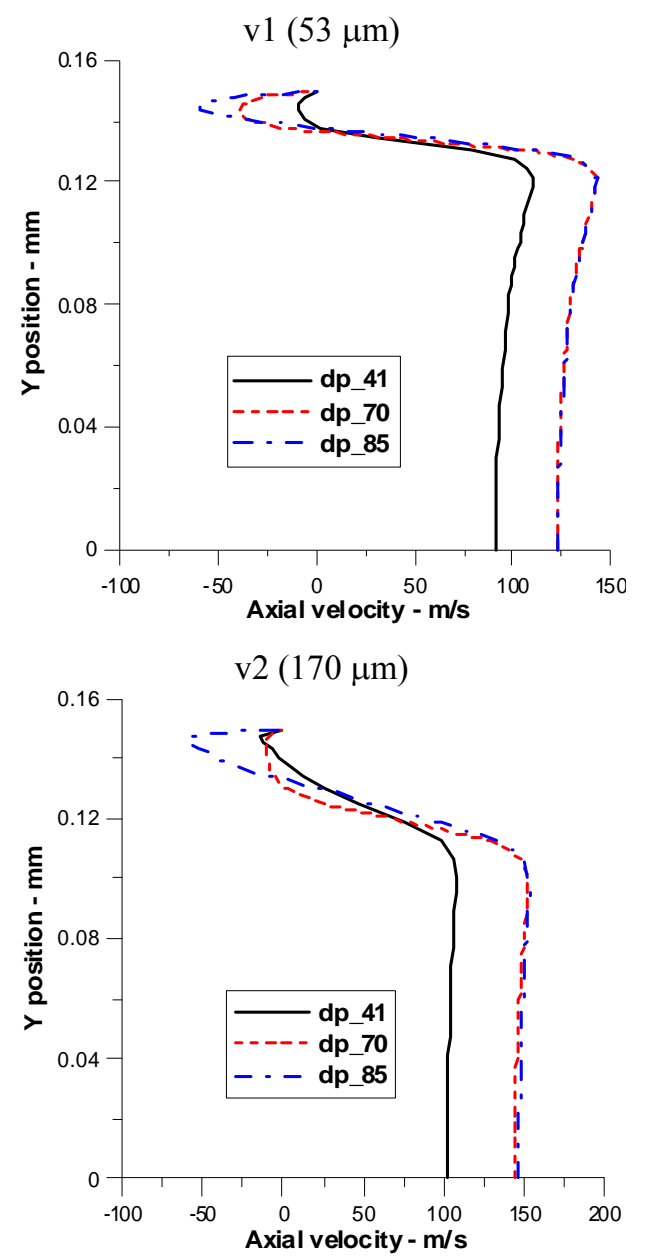

Fig. 9 Velocity profiles in position v1 and v2 at condition without cavitation $(\Delta \mathrm{p}=41$ bar), with moderate cavitation $(\Delta \mathrm{p}=70$ bar $)$ and at choked flow conditions $(\Delta \mathrm{p}=85$ bar $)$.

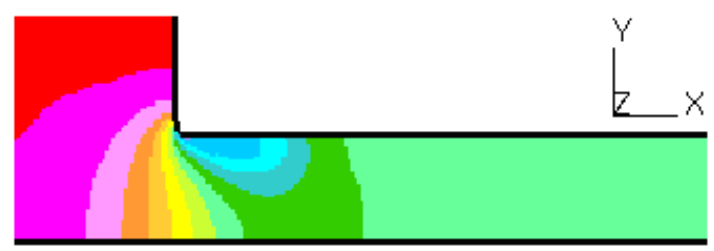

(a)

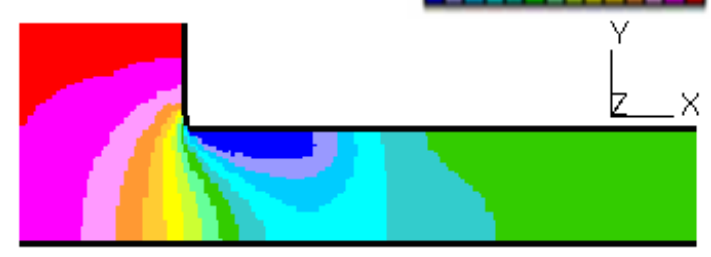

(b)

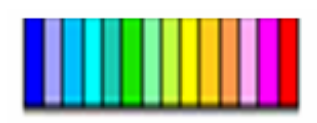

Fig. 10 (a) Pressure distribution at non cavitating conditions $(\Delta \mathrm{p}=58$ bar $)$, and (b) at $\mathrm{CC}(\Delta \mathrm{p}=69$ bar) at longitudinal middle plane. Colour scale: $-5 \mathrm{E}+05-1 \mathrm{E}+07$. 
nozzle flow, as confirmed in Fig. 8: the onset of cavitation appears in the low pressure recirculation area and grows with increasing pressure drop.

\subsubsection{Pressure profiles and distribution}

In Fig. 10 the predicted pressure fields are shown at non cavitating conditions and at critical conditions. The most relevant feature concerns the pressure gradients, which are confined to the entrance area of the nozzle independently of the pressure drop. In agreement with Winklhofer et al. (2001), the solution shows that the main pressure gradient layer covers a size of about one throttle diameter, with half of this layer extended into the upstream area in front of the throttle entrance. The images also illustrate the precise location of the low pressure recirculation zone commented before, where at increasing pressure drop cavitation is initiated. At critical conditions, the minimum pressure predicted at the entrance of the nozzle is less than the saturation pressure, which indicates that cavitation appears in this area (Fig. $10 \mathrm{~b})$. Moreover, there is an inverse relationship between the minimum pressure and the amount of vapor predicted. Indeed, this is verified by examining the effect of nucleus number density on the minimum pressure and the injection rate (see Fig. 5). It is seen that with decreasing minimum pressure, the injection rate decreases, which is consistent with an increase of the predicted volume fraction of vapour.

Fig. 11 illustrates the pressure distribution profile of the simulated case at $\mathrm{CC}$ conditions $(\Delta \mathrm{p}=69$ bar) along the symmetry line of the vertical middle plane compared with the experimental one $(\Delta \mathrm{p}=65$ bar). As already mentioned, the predicted pressure in the inlet is lower than the experimental value due to the pressure loss calculated. Although, the minimum pressure attained in the calculations is smaller than in the experiment, due to the different critical conditions obtained, there is good qualitative agreement. It is seen that along the line the pressure minimum appears in the zone of low pressure recirculation, followed by the pressure recovery downstream of the throttle entrance.

\subsection{Flow field}

Fig. 12 shows contours of velocity and vapour volume fraction, illustrating a pair of vortices with smaller volume fraction of vapor that appear in the middle of the nozzle and extend towards the exit. These predicted vortices are documented in the literature (Peng Kärrholm et al., 2007; Shi

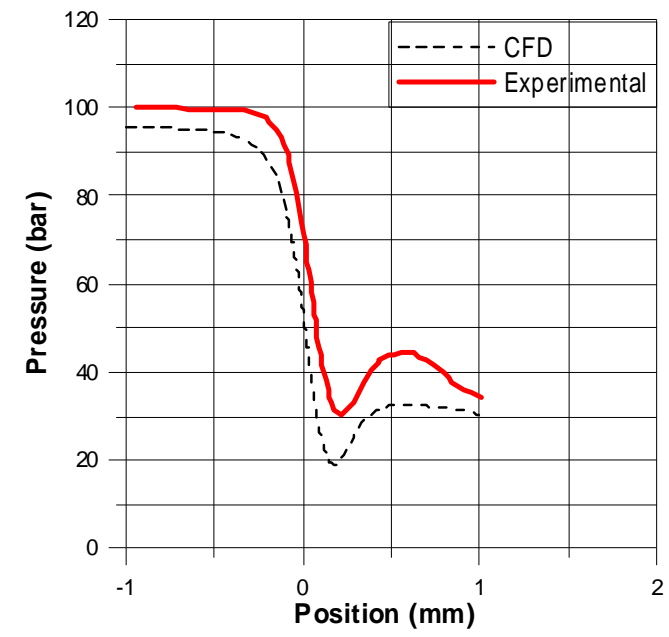

Fig. 11 Predicted and experimental pressure profiles at $\mathrm{CC}$ conditions.

(a)

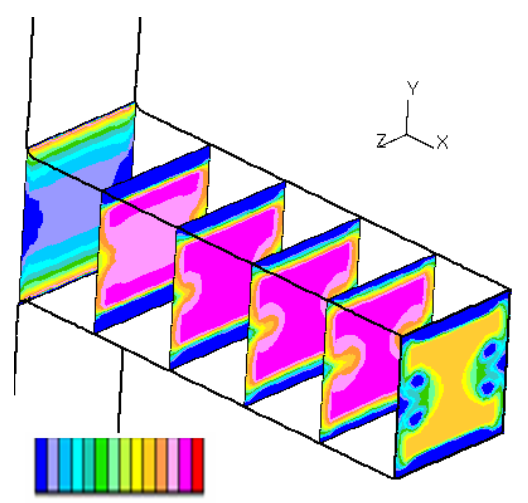

(b)

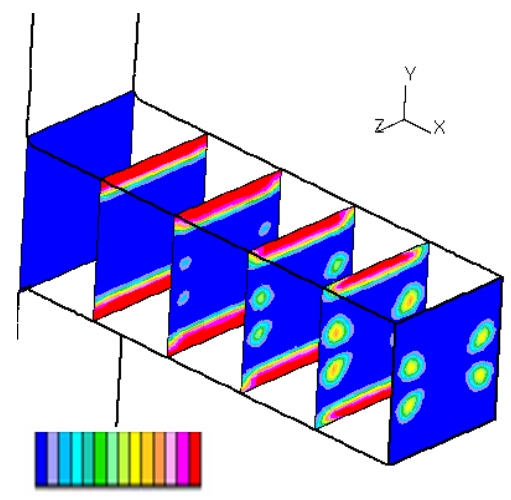

Fig. 12 (a) Velocity magnitude and (b) volume fraction of vapour fields in perpendicular planes to the flow direction. Colour scale: $100-160 \mathrm{~m} / \mathrm{s}$ for the velocity magnitude; $0-1$ for the volume fraction of vapour.

and Arafin, 2010) for this kind of Diesel injectorlike geometries. The domain was recalculated with the whole geometry to discard the possibility that the vortices could be associated with the symmetry boundary condition. As seen in Fig. 12 the flow is accelerated in the upper and lower half of the nozzle where the inlet is rounded, contributing to the formation of vortices in the interior. Calculations made with variants of the k$\varepsilon$ and the k- $\omega$ models predicted vortices at similar 
location and strength but their size depended on the turbulence model and treatment of the near wall flow. Since experimental data is not available to validate the aforementioned results, it is not clear which model is more accurate. Rotational vortices and vortex induced cavitation are typical characteristics in diesel injectors and are known to affect significantly the emerging fuel spray (Roth et al., 2002; Gavaises and Andriotis, 2006).

\section{CONCLUSIONS}

A commercial code (STAR-CD, 2008) has been used to study cavitation flow within an injectorlike geometry. The solution was validated with experimental data documented by Winklhofer et al. (2001). It was found that the cavitation model could predict reasonably well the observed pressure field and low pressure recirculation area linked to the presence of cavitation at the nozzle entrance. Although there is a slight overprediction of the critical conditions pressure drop, the model rendered accurately the effects of pressure drop variation in the nozzle. The amount of vapour generated in the cavitation region is under-estimated by the model. This could be due to the overestimation of the liquid viscosity, the influence of the turbulence model or of the vapour-liquid phase interactions not taken into account.

Vortices are formed inside the nozzle, resulting in localized pockets of low pressure where cavitation regions are detected. Given the insufficiently documented experimental data, the study does not allow safe conclusions to be drawn about the effectiveness of the cavitation model concerning the prediction of the cavitating vortical structures. Hence, it would be very useful to deepen the experimental studies of the flow inside injectors, to provide more precise and extensive validation of the cavitation and turbulence models. Additionally, since the final objective is to simulate diesel injector flow, calculations with higher injection pressures should be performed, as diesel injection pressures are often above 100 Mpa. However, the study presented here confirms that the model is sufficiently accurate and may be used to gain insight into the flow phenomena occurring within a diesel injector.

\section{ACKNOWLEDGEMENTS}

This research has been funded by the Spanish Government in the frame of the Project "Métodos
LES para la simulación de chorros multifásicos", Ref.ENE2010-18542. The authors also acknowledge the financial support of the Universidad Politécnica de Valencia under the contract Reference PAID-2759 and the Generalitat Valenciana, under the contract GV/2010/039.

\section{REFERENCES}

1. Arai M, Shimizu M, Hiroyasu H (1985). Breakup length and spray angle of high speed jet. Proceedings of the 3rd International Conference on Liquid Atomization and Spray Systems (ICLASS), London, IB/4/1-10.

2. Arcoumanis C, Badami M, Flora H, Gavaises M (2000). Cavitation in real-size multi-hole diesel injector nozzles. Society of Automotive Engineers International (SAE) 2000-01-1249.

3. Balasubramanyam MS, Bazarov VG, Chen CP (2010). Numerical design investigation of a hydromechanical pulsator for rocket motor injector dynamics research. Engineering Applications of Computational Fluid Mechanics 4:314-325.

4. Berwerk W (1959). Flow pattern in diesel nozzle spray holes. Proceedings of the Institute of Mechanical Engineers 173(25):655-660.

5. Bierbrauer F, Zhu SP (2008). A numerical model for multiphase flow based on the GMPPS formulation, part II: Dynamics. Engineering Applications of Computational Fluid Mechanics 2:284-298.

6. Brennen CE (1995) Cavitation and Bubble Dynamics. New York, Oxford University Press.

7. Chaves H, Knapp M, Kubitzek A (1995). Experimental study of cavitation in the nozzle hole of diesel injectors using transparent nozzles. Society of Automotive Engineers International (SAE) 950290.

8. Christafakis A, Tsangaris S (2008). Twophase flows of droplets in contractions and double bends. Engineering Applications of Computational Fluid Mechanics 2:299-308.

9. Gavaises M, Andriotis A (2006). Cavitation inside multi-hole injectors for large Diesel engines and its effect on the near-nozzle spray structure. Society of Automotive Enginners International (SAE) 2006-01-1114.

10. He L, Ruiz F (1995). Effect of cavitation on flow and turbulence in plain orifices for highspeed atomization. Atomization and Sprays 5(6):569-584. 
11. Hiroyasu H, Arai M, Shimizu M (1991). Break-up length of a liquid jet and internal flow in a nozzle. Proceedings of the 5th International Conference on Liquid Atomization and Spray Systems (ICLASS). Gaithersburg, MD, 275-282.

12. Joseph DD (1998). Cavitation and the state of stress in a flowing liquid. Journal of Fluid Mechanics 366:367-378.

13. Knapp RT, Daily JW, Hammitt FG (1970). Cavitation. New York; London: McGraw-Hill.

14. Margot X, Hoyas S, Fajardo P, Patouna S (2010). Moving mesh generation strategy solving an injector internal flow problem. Mathematical and Computer Modelling 52(78):1143-1150.

15. Martynov S (2005). Numerical Simulation of the Cavitation Process in Diesel Fuel Injectors. PhD Thesis. University of Brighton.

16. Nurick WH (1976). Orifice cavitation and its effect on spray mixing. ASME Transactions, Journal of Fluids Engineering 98:681-687.

17. Payri F, Margot X, Patouna S, Ravet F, Funk M (2009). A CFD study of the effect of the needle movement on the cavitation pattern of diesel injectors. Society of Automotive Engineers International (SAE) 2009-24-0025.

18. Peng Kärrholm F, Weller H, Nordin N (2007). Modelling injector flow including cavitation effects for diesel applications. 5th Joint ASME/JSME Fluids Engineering Conference. San Diego, California USA.

19. Rayleigh L (1917). On the pressure developed in a liquid during the collapse of a spherical cavity. Phil. Mag. 34:94-98.

20. Roth H, Gavaises M, Arcoumanis C (2002). Cavitation initiation, its development and link with flow turbulence in Diesel injector nozzles. Society of Automotive Engineers International (SAE) 2002-01-0214.

21. Shi JM, Arafin M (2010). CFD investigation of fuel property effect on cavitating flow in generic nozzle geometries. ILASS-Europe 2010. Brno, Czech Republic.

22. Soteriou C, Andrews R, Smith M (1995). Direct injection diesel sprays and the effect of cavitation and hydraulic flip on atomization. Society of Automotive Engineers International (SAE) 950080.

23. Som S, Aggarwal S. K, El-Hannouny E.M, Longman, D.E (2010). Investigation on nozzle flow and cavitation characteristics in a diesel injector. Journal of Engineering for Gas Turbines and Power 132(4):042802-1.

24. STAR-CD (2008). Methodology. V4.06, CD adapco.
25. Winklhofer E, Kull E, Kelz E, Morozov A (2001). Comprehensive hydraulic and flow field documentation in model throttle experiments under cavitation conditions. ILASS-Europe 2001. 2-6 September, Zurich.

26. Yuan W, Sauer J, Schnerr GH (2000). Modelling and computation of unsteady cavitation flows in injection nozzles. 1st International Colloquium on Microhydrodynamics. Paris, France.

27. Yuan W, Schnerr GH (2001) Cavitation in injection nozzles-Effect of injection pressure fluctuations. Proceedings of 4th International Symposium on Cavitation. Pasadena, California, USA. 